\title{
Editorial
}

\section{What is In a Name? Vor a Number?): The updated Enzyme Classifications}

From the Department of Natural Sciences, University of Michigan

Dearborn, College of Arts Sciences and Letters, Dearborn, Michigan

A new enzyme category! Since the first six classes of enzyme categories were approved by the International Union of Biochemistry (IUB), thousands of enzymes have been assigned to the core group of six classifications. The original classification scheme was released as a joint effort of the IUB with the International Union of Pure and Applied Chemistry (IUPAC) to define enzyme catalyzed reactions. This numbered cataloging is the accepted standard. Over time, updated versions of the categories and definitions were released, and a full history of changes and supplements can be found on the IUBMB nomenclature page (https://www.qmul.ac.uk/sbcs/ iubmb/home/WNew.html).

The classifications depicted the enzyme names using a series of four numbers. The first number defines the enzyme category for the reaction type: oxidoreductases, transferases, hydrolases, lyases, isomerases, ligases, and now, translocases (Table I). The second number denotes information about the compounds or groups, while the third specifies the type of reaction. The fourth number is specific, giving each enzyme a number akin to a serial number. The four-number sequence is commonly observed on metabolic charts. A brief review by Tipton and McDonald nicely summarizes the reaction classifications and a full listing is available via the IUBMB web sites[1] (https://www.qmul.ac.uk/sbcs/iubmb/enzyme/rules.html, https:// www.qmul.ac.uk/sbcs/iubmb/enzyme/).

The numbering system worked pretty well but was far from complete. The IUB recognized the importance of genomics and added "molecular biology" to the name and is now called the IUBMB. New technology and techniques enhanced our understanding of the complex mechanisms of membrane transport and the IUBMB noted the prevalence of transport enzymes and added the category EC7 translocases in 2018. EC7 includes several subclasses of proteins responsible for "transfer." This new category simplifies assignments and minimizes the confusing nomenclature of "in and out" and "cis and trans." An example is demonstrated for EC 7.3.2.1 (Fig. 1) [2, 3]. The translocases are defined as:

Volume 47, Number 4, July/August 2019, Pages 481-483

*To whom correspondence should be addressed.

E-mail:marilee@umich.edu

Received 21 February 2019; Accepted 7 April 2019

DOI 10.1002/bmb.21251

Published online 7 May 2019 in Wiley Online Library

(wileyonlinelibrary.com)
EC 7.1 Catalyzing the translocation of hydrons

EC 7.2 Catalyzing the translocation of inorganic cations

EC 7.3 Catalyzing the translocation of inorganic anions and their chelates

EC 7.4 Catalyzing the translocation of amino acids and peptides

EC 7.5 Catalyzing the translocation of carbohydrates and their derivatives

EC 7.6 Catalyzing the translocation of other compounds

In addition to the number, enzymes have names that can be systematic or descriptive and the common name is usually provided. These trivial names are often created by researchers but only the IUBMB can assign the number. For a brief summary of IUBMB nomenclature, see the IUBMB News Issue 6 , 2018. In this online issue, Keith Tipton describes the new categories and compares the translocases to those membrane proteins that transport without enzymatic reaction (https:// iubmb.org/newsletter/).

But what if you find the EC classification useful but limited? You are not alone! Although lauded and critical, the IUBMB system is imperfect and numerous biochemists bemoaned the lack of clarity as well as the inconsistency. While the system neatly categorizes according to the reaction type, it is not as useful in decoding mechanisms, substrates, products, or other parameters used to identify and compare reactions. New databases have been created to alleviate confusion and clarify reactions. The largest, the BRaunschweig ENzyme DAtabase (BRENDA), annotates millions of bits of curated data on enzyme-catalyzed reactions, including substrate and products, inhibitors, cofactors, kinetic data, synonyms and species-specific information (http://www.brenda-enzymes.org/) [4]. Other systems have proven useful to related areas of research; biologists often use the Kyoto Encyclopedia of Genes and Genomes (KEGG) database, which provides reaction data in a distinct format and reaction chemistry, and this is examined in a review of the numerous enzymes that are linked to varying reactions in other databases [5].

Researchers have crafted processes for automatic assignment of the enzyme numbers, and attempted to indicate the large numbers of reactions that remain categorized as "orphans" [6]. Their approaches include annotation for atom coding, structural examples of reactions, and the system considers other factors-such as how to differentiate a 


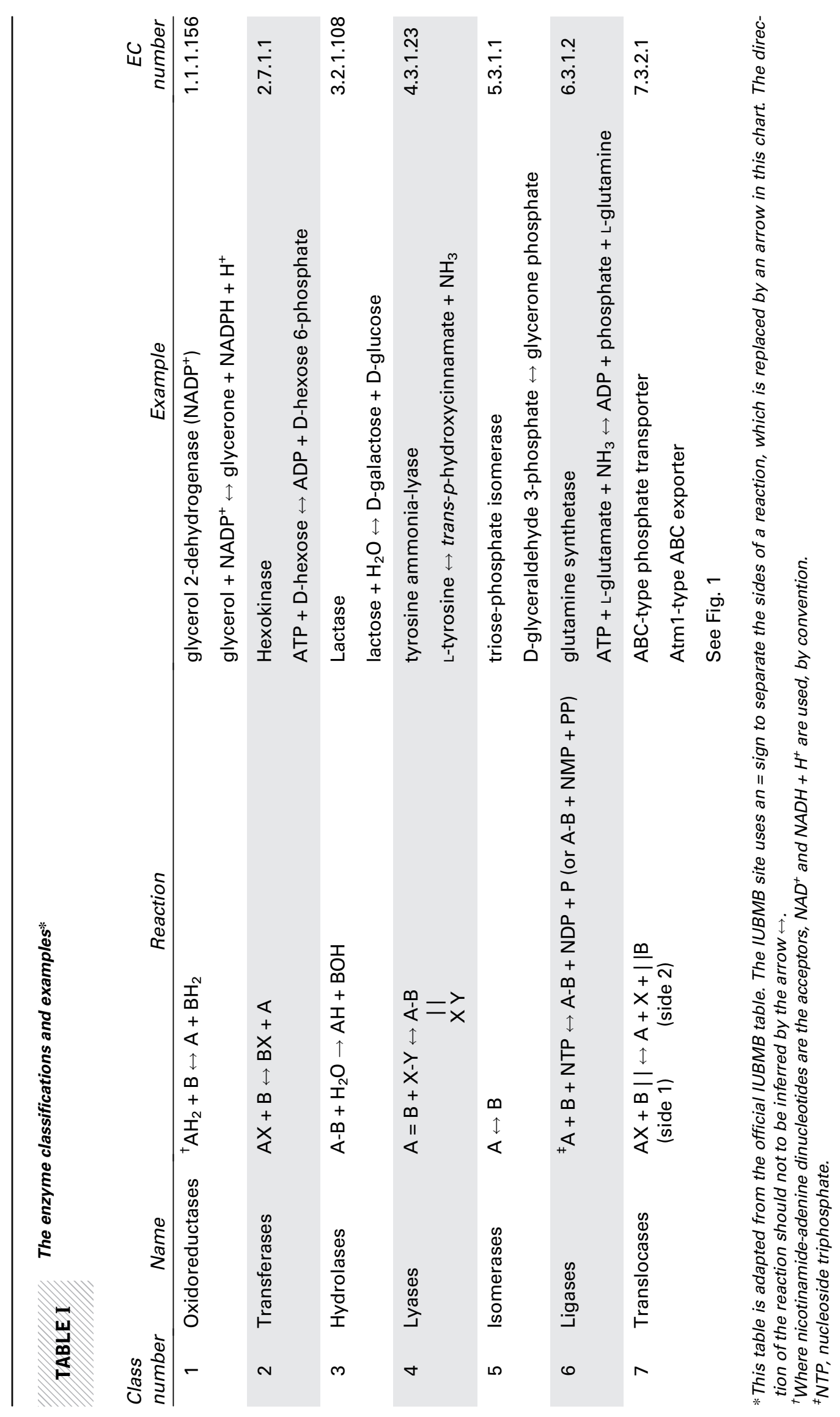



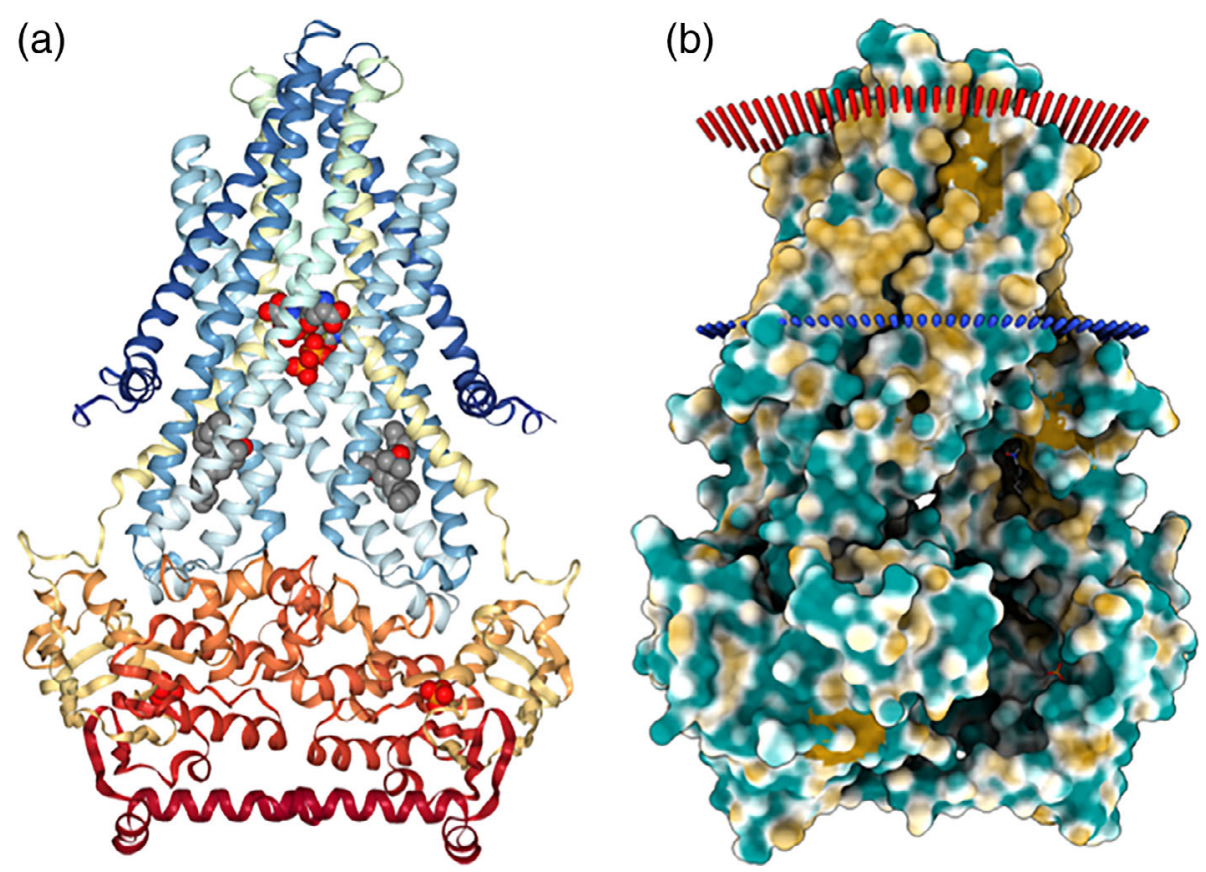

FIG 1

Atm1-type ABC exporter EC 7.3.2.1 An example of a translocase, of the category EC 7.3: Catalyzing the translocation of inorganic ions and their chelates, and linked to the hydrolysis of a nucleoside transporter (EC 7.3.2), is a member of a super family of ATP binding cassette $(A B C)$ transport proteins: It moves the molecule from one membrane side to the other of the membrane [3].

ATP $+\mathrm{H}_{2} \mathrm{O}+$ phosphate-[phosphate-binding protein][side 1] $\leftrightarrow$ ADP + phosphate + phosphate[side 2] + [phosphate-binding protein][side 1]. (A) 4MRV shown in chainbow format. (B) Surface ligand lipophilicity (open source copyright free www. wwpdb.org https://www.rcsb.org/structure/4mrv). [Color figure can be viewed at wileyonlinelibrary.com]

coenzyme such as NADH from another ligand, or how to include these as "substrate/product pairs" (https://www. genome.jp/kegg/). The NCBI ENTREZ attempts to link all data including nucleotide and amino acid sequences, taxonomy and structures (https://www.ncbi.nlm.nih.gov). Many of these other databases are conveniently linked from the IUBMB website descriptions of each enzyme. From the IUBMB enzyme database home page, you can click on the number, which takes you to a page describing the reaction, accepted name, and references. All the enzymes can be searched at http://www.enzyme-database.org/class.php. A guide to using the new database can be found at https://iubmb.org/a-briefguide-to-enzyme-classification-and-nomenclature-rev/.

The addition of a new category will impact the many textbooks that will now need to be updated to reflect these changes. However, faculty and students can easily access the information to rapidly update lectures and notes. Table I shows the simplicity of the classes of the reactions and can be downloaded and provided as a supplement.

\section{REFERENCES}

[1] Tipton, K. \& McDonald, A. (2018) A brief guide to enzyme nomenclature and classification. https://iubmb.org/a-brief-guide-to-enzyme-classification -and-nomenclature-rev/

[2] Cuthbertson, L., Kos, V., Whitfield, C. (2010) ABC transporters involved in export of cell surface glycoconjugates. Microbiol. Mol. Biol. Rev. 74, 341-362.

[3] Lee, J. Y., Yang, J. G., Zhitnitsky, D., Lewinson, O., Rees, D. C. (2014) Structural basis for heavy metal detoxification by an Atm1-type ABC exporter. Science 343(80), 1133-1136.

[4] Placzek, S., Schomburg, I., Chang, A., Jeske, L., Ulbrich, M., Tillack, J., Schomburg, D. (2017) BRENDA in 2017: new perspectives and new tools in BRENDA. Nucleic Acids Res. 45, D380-D388.

[5] Dönertaş, H. M., Cuesta, S. M., Rahman, S. A., Thornton, J. M. (2016) Characterising complex enzyme reaction data. PLoS One 11, 1-18.

[6] Egelhofer, V., Schomburg, I., Schomburg, D. (2010) Automatic assignment of EC numbers. PLoS Comput. Biol. 6, e1000661. https://doi.org/10. 1371/journal.pcbi.1000661. 